\title{
Pratiques agricoles pour la réduction des produits phytosanitaires. le rôle de l'apprentissage collectif
} Adoption of pesticides reducing agricultural practices. The role of collective learning

Geneviève Nguyen, Jean-Pierre Del Corso, Charilaos Képhaliacos et Henri Tavernier

\section{(2) OpenEdition}

\section{Journals}

Édition électronique

URL : http://journals.openedition.org/economierurale/3817

DOI : 10.4000/economierurale.3817

ISSN : 2105-2581

Éditeur

Société Française d'Économie Rurale (SFER)

Édition imprimée

Date de publication : 15 février 2013

Pagination : 105-121

ISSN : 0013-0559

Référence électronique

Geneviève Nguyen, Jean-Pierre Del Corso, Charilaos Képhaliacos et Henri Tavernier, « Pratiques agricoles pour la réduction des produits phytosanitaires. le rôle de l'apprentissage collectif ",

Économie rurale [En ligne], 333 | janvier-février 2013, mis en ligne le 15 février 2015, consulté le 30 avril 2019. URL : http://journals.openedition.org/economierurale/3817 ; DOI : 10.4000/

economierurale.3817 


\title{
Pratiques agricoles pour la réduction des produits phytosanitaires Le rôle de l'apprentissage collectif
}

\author{
Geneviève NGUYEN • Université de Toulouse, INP-ENSAT, Castanet-Tolosan, \\ nguyen@ensat.fr \\ Jean-Pierre DEL CORSO, Charilaos KEPHALIACOS, Henri TAVERNIER • Université de Toulouse, \\ ENFA, Castanet-Tolosan
}

En partant d'une expérience multi-partenariale originale de MAET-DCE portée par une coopérative, ce travail se propose d'analyser le rôle de l'apprentissage collectif sur la réduction des risques liés à l'adoption de pratiques agricoles alternatives. Le processus de transmission de l'information technique ainsi que les formes de coordination associées ont été analysés en mobilisant des approches principalement de l'économie institutionnelle traitant de l'incertitude et des modalités actuelles du conseil en agriculture. L'analyse montre que l'action de la coopérative est au centre de la création conjointe de plusieurs catégories de biens, reposant sur différents types de transaction informationnelle.

MOTS-CLÉS : Apprentissage collectif, Conseil, Coûts de transaction, Incertitude, Mesures agri-environnementales, Produits phytosanitaires, Bassin versant Adour-Garonne

\begin{abstract}
Adoption of pesticides reducing agricultural practices. The role of collective learning Based on an original multi-partnership experience of agri-environmental measure (MAET$D C E)$ carried by a cooperative, this study aims to analyse the role of collective learning on the reduction of risks associated with the adoption of alternative agricultural practices. The process of transmission of technical information and the associated forms of coordination have been analysed using a conceptual framework inspired by works done in institutional economics on uncertainty and extension service in agriculture. The analysis shows that the cooperative is at the center of the joint production of different categories of goods, based on different types of informational transactions. (JEL: Q53, Q16, Q18, Q52)
\end{abstract}

KEY-WORDS: Collective Learning, Extension service, Transaction Costs, Uncertainty, Agri-environmental Measures, Pesticides, the Adour-Garonne River Basin

C omme en témoignent la Directive cadre sur l'eau (DCE) de 2000 modifiée en 2008, le dernier bilan santé de la PAC et les engagements du Grenelle de l'environnement de 2008, la qualité de l'eau est devenue un des objectifs prioritaires de l'Union européenne. En accord avec cet objectif, le dernier rapport d'expertise de l'INRA-CEMAGREF met en avant l'intérêt d'un référencement des pratiques agricoles alternatives en matière d'emploi de produits phytosanitaires. Il souligne également l'importance de prendre en compte les conditions d'adoption et de mise en œuvre de ces pratiques tant au niveau de l'exploitation agricole qu'au niveau de son environnement économique et social.

Notre étude s'inscrit dans le cadre d'un projet de recherche PSDR/CCRDT MidiPyrénées axé sur l'analyse des conditions d'adoption de techniques innovantes en matière d'utilisation de produits phytosanitaires en agriculture. Cette adoption dépend notamment de la capacité de l'agriculteur à surmonter les risques et les incertitudes liés à un changement de pratiques et à utiliser pour cela différentes ressources matérielles et immatérielles. Dans cet article, nous centrons particulièrement notre attention sur l'information en tant que ressource 
immatérielle mobilisable par l'agriculteur pour réduire les risques et les incertitudes. L'information utilise plusieurs supports et véhicules: messages techniques des conseillers agricoles, échanges entre agriculteurs, recommandations inclues dans des cahiers des charges, etc. La transmission et l'acquisition de cette information génèrent des coûts d'apprentissage. Ceuxci sont supportés soit par l'agriculteur, soit par des acteurs collectifs privés ou publics. Dès lors, quelles peuvent être les modalités de partage des coûts entre ces catégories d'acteurs? Comment peut-on apprécier l'efficacité globale d'un dispositif d'information?

Dans cet article, nous commençons par présenter la problématique de la gestion de la qualité de l'eau telle qu'elle se pose dans le bassin versant Adour-Garonne support à notre étude. Un retour sur la littérature économique nous permettra ensuite de clarifier les déterminants de l'adoption de nouvelles pratiques agricoles et de préciser le rôle dévolu à l'information technique. Cela nous conduira à mobiliser le concept de «trans-actions» de Commons (1931) et à proposer une nouvelle interprétation de la notion de coûts de transaction appliquée à l'analyse du processus de «transaction informationnelle ». Enfin, nous mobiliserons ce cadre conceptuel pour étudier le processus d'adoption d'une Mesure agri-environnementale territorialisée s'inscrivant dans la Directive cadre sur l'eau (MAET-DCE), par les adhérents d'une coopérative localisée sur le bassin versant Adour-Garonne. Nous analyserons plus précisément: (i) les différentes formes de coordination et leurs impacts sur les coûts de transmission et d'apprentissage de nouvelles pratiques; (ii) la relation entre, d'une part, l'accès à l'information et sa qualité, et d'autre part le risque d'adoption; (iii) les modalités pratiques de prise en charge des coûts ex-ante d'adoption entre les «trans-actants».

\section{État des lieux de l'adoption des pratiques alternatives}

\section{Pollution de l'eau et réponses en matière de politiques publiques}

Le bassin versant Adour-Garonne s'étend sur deux régions entières, Aquitaine et Midi-Pyrénées et comprend 120000 km de cours d'eau. Outre le problème de déficit structurel en eau, le bassin versant AdourGaronne est confronté à un grave problème de qualité de l'eau. Si les pollutions domestiques et industrielles semblent être aujourd'hui sous contrôle, la pollution diffuse des eaux de surface et souterraine par l'activité agricole persiste. La plupart des matières actives détectées dans les rivières du bassin versant entrent dans la composition des molécules de produits phytosanitaires utilisées en grandes cultures (Alachlore, Benoxacor, Bentazone, Isoxaflutole, etc.). Les observations de terrain montrent que les pratiques sont encore insuffisamment raisonnées (peu de désherbage mécanique, rotations courtes, applications de doses de désherbage maximales et trop tardives, etc.). Cette pollution diffuse par l'agriculture devient d'autant plus inquiétante qu'elle menace désormais un certain nombre de points de captage en eau potable et qu'elle compromet la sauvegarde d'écosystèmes aquatiques remarquables.

Pourtant, dès le début des années 1990, d'importants moyens financiers et logistiques ont été mobilisés dans le bassin versant Adour-Garonne comme dans les autres régions françaises: mise en place de groupes régionaux d'action phytosanitaire, définition de schémas directeurs d'aménagement et de gestion des eaux, adoption d'une démarche de zonage, de surveillance de la qualité de l'eau etc. Le principe «pollueur-payeur» a par ailleurs été affirmé et son champ d'application étendu. Parallèlement, une politique d'incitation contractuelle visant à modifier les pratiques agricoles sur le long terme a vu le jour à travers la mise en place de 
MAET-DCE. Dans la Région Midi-Pyrénées, les MAET-DCE ont bénéficié d'un co-financement important de la part du FEADER et de l'Agence de l'eau AdourGaronne. Malgré ces efforts, les résultats obtenus sont très nuancés.

\section{Une expérience originale de MAET-DCE portée par un acteur privé}

Des expériences originales ont toutefois été initiées et semblent davantage porter leurs fruits. C'est par exemple le cas, en MidiPyrénées, d'un partenariat multi-acteurs qui a pris naissance au début des années 1990 autour de l'organisation de la surveillance biologique du territoire. À côté d'acteurs classiques - Service régional de protection des végétaux (SRPV, service de la DRAAF), Chambres d'agriculture et Instituts techniques - ce partenariat concerne des opérateurs privés, parmi lesquels une coopérative localisée sur le bassin versant Adour-Garonne, au centre de notre étude. Cette coopérative de taille moyenne, spécialisée dans les grandes cultures travaille avec plus de 1500 agriculteurs exploitant plus de 45000 ha. Elle propose à ses adhérents des services de collecte, d'approvisionnement en intrants, de fabrication d'aliments du bétail, etc. Elle a par ailleurs initié diverses démarches contractualisées de qualité et a participé à la mise en œuvre de CTE collectifs. Ce partenariat entre le SRPV/DRAAF et la coopérative s'est renforcé depuis que cette dernière a pris l'initiative de porter en 2009 un projet MAETDCE.

Le projet de MAET dont il est ici question est axé sur un objectif unique de réduction des produits phytosanitaires utilisés en grandes cultures. Ce programme de réduction progressive du nombre de doses homologuées de traitements herbicides et non herbicides bénéficie d'un dispositif d'accompagnement. Celui-ci prend notamment la forme d'un suivi individuel réalisé actuellement par une équipe de six techniciens de la coopérative ${ }^{1}$. Chacun de ces techniciens rend des visites régulières aux exploitants (dix par an), au cours desquelles il aide l'agriculteur à raisonner ses pratiques phytosanitaires. Des actions d'animation collective sur le terrain telles des visites en groupes restreints de champs d'essais variétaux, de réunions bilan sur les résultats collectifs obtenus, etc., complètent le dispositif d'accompagnement piloté par la coopérative.

L'engagement d'une coopérative dans une MAET-DCE peut paraître paradoxal dans la mesure où celle-ci propose à ses adhérents un service classique d'approvisionnement. Il l'est moins si l'on considère, d'une part que le plan Ecophyto 2018 entre dans une phase d'opérationnalisation décisive et d'autre part, que le modèle professionnel du conseil en agriculture est en cours de réexamen (Rémy et al., 2006). Ainsi, grâce à sa participation à la MAET, la coopérative peut être en mesure d'anticiper les bouleversements futurs de son activité.

Nous examinons maintenant les enjeux d'un changement de pratiques pour les agriculteurs. Nous le faisons en clarifiant les concepts de risque et d'incertitude et en explicitant les modes de coordination sous-jacents aux apprentissages.

\section{Risques/incertitudes de l'adoption et processus d'apprentissage}

\section{Les mesures de politique environnementale appréhendées comme des facteurs d'incertitude}

Bien que nombreux, les risques en agriculture sont relativement bien identifiés et étudiés : (risque de variation de la production, risque de fluctuations des prix, risque de changements de politique publique). Le concept d'aversion aux risques permet par ailleurs de les prendre en compte dans le

1. À terme, il est prévu que cette équipe comprenne douze techniciens. 
processus de prise de décision des agriculteurs. Ainsi, le recours à des politiques de compensation ou à des pratiques assurancielles est un moyen pour faire face à l'avènement de phénomènes dont la probabilité est connue (Just et Pope, 1979 ; Binswanger, 1980 ; Hardaker, 2000 ; Hardaker et al., 2004). Relevant de la théorie de l'utilité espérée, le concept d'aversion est donc parfaitement compatible avec une logique d'optimisation sous contraintes. Malgré ses lacunes, il reste un outil économique essentiel pour appréhender et gérer le risque.

Cependant, avec l'émergence des problèmes environnementaux en agriculture, il n'est plus seulement question de risques mais aussi d'incertitudes. L'étroite interdépendance entre les facteurs physico-chimiques, écologiques, techniques et socio-économiques s'accompagne d'une complexification des situations à traiter. Face à elle, il est impossible de déterminer avec certitude les relations de cause à effet entre des pratiques agricoles et le niveau de pollution des eaux superficielles constaté à un moment donné (MEDD, 2005). Pindyck (2007), Godard (1994, 2003) et Vatn (2005) montrent comment, dans un tel contexte, la mise en œuvre de politiques publiques en matière de gestion des ressources naturelles et de l'environnement devient particulièrement délicate car il n'est pas possible d'évaluer de manière simple et objective les coûts et bénéfices d'une politique. En effet, au-delà de la complexité des phénomènes biologiques et physico-chimiques en jeu, les incertitudes sont exacerbées par la présence de problèmes d'irréversibilité, par l'inscription dans un horizon temporel long et par la nécessité de considérer les critères de justice et d'équité de l'action publique. Pour améliorer les approches classiques de politiques publiques, ces auteurs soulignent ainsi l'importance de préciser la frontière entre risques et incertitudes, mais aussi la nature des incertitudes et leur dynamique.
Pour y parvenir, nous recourons à la typologie des incertitudes proposée par Callon et al. (2001). Selon ces auteurs, une première situation d'incertitude réside dans le fait que les conséquences sont observables mais les causes difficiles à identifier. Il est cependant possible d'élaborer des scenarii fictifs socialement acceptables par l'ensemble des acteurs. Une deuxième situation d'incertitude est caractérisée par une connaissance en général imparfaite des relations de cause à effet. Des approches inductives peuvent être mobilisées pour relier deux faits observés. Dans ces deux premiers cas, le principe de précaution peut être invoqué. Callon et al. (2001) identifient un troisième type d'incertitudes, dit « radical», et correspondant à un état d'ignorance totale sur la survenance d'événements. Seule une observation a posteriori permet le repérage de l'incertitude radicale ${ }^{2}$.

Cette typologie des incertitudes nous conduit alors à appréhender le phénomène de pollution diffuse des eaux superficielles par l'agriculture comme un état intermédiaire entre une situation de «soupçon» et une situation de «présomption» (ibid.). Face à ce type de situation tout raisonnement fondé sur l'optimisation et le calcul probabiliste est impossible. Dès lors, il devient nécessaire de recourir à d'autres approches pour aborder l'action en situation incertaine, et notamment pour appréhender la mise en œuvre des mesures de politique environnementale.

La mise en œuvre de la politique environnementale tout comme celle des politiques classiques d'orientation de la production

2. La typologie des incertitudes proposée par Callon (1999) est assez proche de celle retenue plus récemment par Vatn (2005). Sur la base de critères somme toute comparables à ceux de Callon, Vatn opère une distinction entre risque ordinaire, incertitude simple et incertitude radicale. Selon cet auteur, l'incertitude radicale est caractéristique de situations dans lesquelles les résultats ne sont ni probabilisables, ni connus. 
agricole fait courir aux agriculteurs un certain nombre de risques en termes de rendement et de revenu. Ces risques, en tant qu'éléments mesurables, peuvent être réduits grâce à des aides publiques permettant de couvrir une partie du coût des investissements engagés et de compenser les pertes financières dues à un changement de pratiques. Toutefois, la politique environnementale se distingue nettement des politiques classiques d'orientation de la production agricole par le fait que les mesures agri-environnementales sont non seulement porteuses de risques, mais aussi de nombreuses incertitudes telles que nous venons de les définir. Ces incertitudes sont liées à la fois au phénomène de pollution diffuse et aux effets à court et long terme des solutions de remédiation proposées.

Face à ces incertitudes, la MAETDCE, considérée dans ce travail, ne peut apporter que des solutions partielles au problème. Au mieux, elle peut conduire à une réduction du niveau de pollution, au pire, elle peut entraîner une dégradation de la situation initiale compte tenu du fait que, comme nous l'avons dit, on ne peut a priori identifier une incertitude radicale liée aux pratiques alternatives préconisées. La question qui demeure est alors celle de la mobilisation des agriculteurs autour d'un dispositif de politique publique imprégné d'incertitudes et reposant sur une double innovation technique (nouvelles pratiques) et institutionnelle (montage multi-partenarial porté par un acteur privé). La gestion de l'information au sein du collectif impliqué dans cette MAET mérite d'être abordée car elle est au centre de cette innovation.

\section{Coordination des acteurs et processus d'apprentissage collectif en situation d'incertitude}

Dans cette étude, nous nous intéressons plus particulièrement au rôle de l'information sur le processus d'adoption des MAET-DCE. Considérant la dimension incertaine de ce dispositif, nous formulons l'hypothèse que l'information, tant dans son contenu que dans la manière dont elle a été construite et véhiculée, est un élément déterminant pour réduire l'incertitude et favoriser l'adoption de nouvelles pratiques agricoles. Si les aides constituent un levier efficace pour limiter les risques en termes de rendement et de revenu (Ghali et al., 2009), elles peuvent ne pas être suffisantes en présence d'incertitudes. Dans cette situation, toute la difficulté est de convaincre l'agriculteur de contractualiser la mesure.

En nous appuyant sur les contributions de Bromley (2006, 2008), nous considérons ici que les agriculteurs ne pourront être convaincus de l'intérêt de changer de pratique sans qu'intervienne un processus de restructuration de leurs croyances et de leurs raisons d'agir. Selon cette perspective, les croyances permettent de rendre une action rationnelle sous une certaine description. Sous-jacentes à la rationalité de l'acteur, elles lui fournissent une représentation de ce qu'il est raisonnable d'entreprendre en certaines circonstances. En leur qualité de croyances établies (ibid.), elles contribuent à mettre fin au doute et sont à l'origine de règles d'action dont se servent les individus pour surmonter les situations d'incertitude radicale auxquelles ils font face.

L'adoption de nouvelles pratiques agricoles correspond à un changement de paradigme pour l'agriculteur et présuppose de ce fait une remise en cause de ses croyances ancrées. Ainsi, la réussite de la MAET-DCE est conditionnée par une reconfiguration individuelle et collective des croyances établies. Cette reconfiguration est essentielle pour passer, au sens de Callon et al. (2001) du « soupçon» à la «présomption », puis à une situation où le raisonnement probabiliste et la construction de normes collectives deviennent à nouveau possibles.

Les enjeux liés à l'adoption de nouvelles pratiques, tels que nous venons de 
les décrire, appellent un nouveau regard sur l'activité de conseil en agriculture. Comme le mettent en évidence les travaux d'Hélène Brives (2006), la prise en compte des questions environnementales s'accompagne d'une remise en question des compétences du conseiller et de son champ d'action. Au-delà de la prestation de service, il s'agirait pour le conseiller en agriculture d'initier une véritable démarche de «co-construction» de connaissances et de pratiques. Par ce travail, les conseillers participeraient à déplacer les résistances et à faire évoluer les croyances et les incertitudes.

Dans notre étude, nous proposons d'apporter un éclairage complémentaire à celui de Brives en précisant les modalités de coordination qui englobent les relations entre l'agriculteur et le conseiller agricole. Selon nous, la manière dont l'agriculteur modifie ses croyances établies et devient ainsi convaincu du bien-fondé d'un changement de pratiques, ne peut être compris que si le changement des règles d'action est appréhendé dans sa globalité. Au-delà de la relation directe entre l'agriculteur et le conseiller, il est important d'analyser comment, au sein du dispositif multi-partenarial MAET-DCE, s'effectuent les transferts des droits d'usage sur les ressources concernées (ressources en eau et informationnelles) et la nouvelle répartition des coûts de transaction associés. Nous définissons ces coûts comme la somme des coûts directement liés à la contractualisation des mesures (investissement en machines et en main-d'œuvre), et ceux liés à la réduction des risques, au déplacement des incertitudes et à leur internalisation partielle (en référence à Callon, 1999). Pour notre analyse des modalités de coordination, nous mobiliserons le concept de «trans-action» de Commons (1931). La trans-action, littéralement «l'action entre les hommes », est une activité sociale de négociation de règles communes d'action.
Nous adoptons ainsi une approche de la notion de transaction plus étendue que celle couramment retenue par les économistes néo-institutionnalistes. Plus adaptée à notre questionnement, l'approche de Commons nous permet d'analyser les processus de formation d'un ordre social en considérant les conditions requises pour dépasser une situation conflictuelle (débat/ controverse sur le lien entre les pratiques et le niveau de pollution) et pour favoriser une coopération entre acteurs (apprentissage collectif).

\section{Perception des risques et des incertitudes par les agriculteurs}

Les résultats présentés dans cette section s'appuient sur une première série d'enquêtes exploratoires réalisées entre mai et septembre 2009, auprès d'acteurs institutionnels (DRAAF Midi-Pyrénées, Agence de l'eau Adour-Garonne, Chambres d'agriculture), des techniciens de la coopérative (conseillers, vendeurs) impliqués dans la mise en œuvre du projet MAET-DCE, de 13 agriculteurs engagés dans le projet (sur la quarantaine de contractants) et de 4 agriculteurs non engagés mais adhérents de la coopérative. Les agriculteurs enquêtés ont été choisis aléatoirement à partir d'une liste fournie par la coopérative.

\section{Le choix d'une méthode d'enquête et d'analyse qualitative}

Ces enquêtes ont consisté en des entretiens individuels d'une durée approximative d'une à deux heures. Pour permettre l'expression la plus libre possible des interviewés, nous avons eu recours à un questionnaire combinant à la fois des questions ouvertes et des questions fermées permettant une évaluation psychométrique de leur appréciation du sujet. Notre premier objectif était, en effet, de comprendre les choix d'action des différents acteurs impliqués en accédant, pour cela, à leurs raisons d'agir (i.e. leur engagement ou leur 
refus d'engagement dans la MAET-DCE). Seule une enquête qualitative pouvait nous permettre un tel accès. Notre deuxième objectif était de construire une échelle d'appréciation de certaines perceptions, celle portant sur les risques et incertitudes, celle portant sur les coûts et bénéfices des changements induits, et celle portant sur l'utilité des dispositifs d'information et de conseil.

Les entretiens avec les acteurs institutionnels ont porté sur le montage institutionnel du projet, sur son organisation et sur les résultats escomptés par la MAETDCE. Ceux avec les techniciens de la coopérative ont concerné les changements induits par le dispositif au niveau de leurs relations avec les agriculteurs (objectifs et contenu des visites et des messages). Les entretiens avec les agriculteurs ont eu pour objectif :

- d'identifier les raisons de la contractualisation ou non contractualisation ;

- de repérer les changements techniques et organisationnels induits par la contractualisation et les freins à ces changements ;

- d'évaluer les coûts et les bénéfices directs et indirects liés aux changements ;

- de recueillir les avis des exploitants agricoles sur les différents types de conseil et leur relation à la coopérative.

L'ensemble de ces entretiens a fait l'objet d'une transcription intégrale. Exploité de façon exhaustive, ce matériau nous a permis, par la suite, de recenser les différentes perceptions des agriculteurs visà-vis des risques et des incertitudes, d'en dresser deux typologies (typologie des risques et incertitudes, et typologie des agriculteurs au regard de leur attitude face aux nouvelles pratiques), de repérer les différentes formes de trans-action informationnelle et, in fine, d'analyser l'impact de celles-ci sur la réduction des risques et des incertitudes. C'est également sur la base de ce matériau que nous avons cherché à formuler des hypothèses de travail et des propositions de nature générique visant à renforcer l'efficacité des mesures publiques agro-environnementales.

\section{Objectifs fixés par la MAET-DCE et perceptions des agriculteurs}

Dans le cadre de la MAET-DCE, les objectifs fixés par la contractualisation sont ceux de l'Indicateur de fréquence de traitement cible (IFT), élément stratégique de gestion des risques et incertitudes par la coopérative et les agriculteurs. Après d'âpres négociations entre acteurs institutionnels deux méthodes complémentaires de calcul de l'IFT sont utilisables :

- une prise en compte de l'ensemble du système de cultures de l'exploitation ;

- un calcul d'un IFT moyen sur 5 ans, avec des objectifs de réduction augmentant progressivement à partir de l'année 2 .

Cette deuxième stratégie de calcul de l'IFT permet aux agriculteurs de lisser les risques de non atteinte de l'objectif sur l'ensemble de l'exploitation et sur toute la durée du contrat. Un agriculteur n'ayant pas atteint les objectifs une année ne sera pas pénalisé s'il a pris de l'avance ou s'il s'engage à réaliser plus d'efforts l'année suivante. Il est important de rappeler que l'objectif premier de la coopérative reste la fidélisation de ses membres adhérents en leur proposant un service nouveau en réponse à l'évolution du contexte institutionnel (supra). Son souci est par conséquent de favoriser l'adhésion d'un maximum d'agriculteurs à la MAET en évitant de leur imposer d'emblée des contraintes trop fortes. L'énoncé suivant d'un technicien de la coopérative met en évidence la prise en compte de cette préoccupation :

« La Chambre d'agriculture est partie des baisses de réduction de produits par culture alors que c'est pas comme ça qu'il faut faire. Il faut un raisonnement global 
par exploitation. C'est sûr que si on le fait par culture, rien de tel pour faire peur. »

(Technicien de la coopérative enquêté)

Le tableau 1 ci-dessous résume les événements probables et/ou incertains identifiés par les agriculteurs comme ayant eu (cas des agriculteurs déjà engagés) ou pouvant avoir (cas des agriculteurs non engagés) une influence sur leur décision de contractualisation. Parmi ces événements, nous distinguons ceux qui relèvent davantage du risque mesurable de ceux qui relèvent du domaine des incertitudes. Cette première distinction s'est appuyée sur l'existence ou non d'informations objectives et scientifiques sur le phénomène en question, qui sont véhiculées par les différentes sources d'information disponibles. Une deuxième distinction a trait à la nature même de l'événement: agronomique ou économique. Cette typologie sert à évaluer la manière dont ces risques et/ou incertitudes auront été pris en compte ou non préalablement dans les termes du contrat.

Tableau 1. Risques et incertitudes perçus par les agriculteurs engagés et non engagés dans la MAET-DCE

\begin{tabular}{|c|c|c|}
\hline & Risques & Incertitudes (du soupçon à la présomption) \\
\hline $\begin{array}{c}\text { De nature } \\
\text { agronomique }\end{array}$ & $\begin{array}{l}\text { - Diminution du rendement } \\
\text { et de la qualité (mycotoxines). }\end{array}$ & $\begin{array}{l}\text { - Effets des pratiques préconisées (TSL, semis tardif) } \\
\text { sur le salissement des parcelles et l'érosion des sols. } \\
\text { - Apparition de résistances liées à l'utilisation d'un } \\
\text { nombre limité de matières actives à spectre large. }\end{array}$ \\
\hline $\begin{array}{l}\text { De nature } \\
\text { économique }\end{array}$ & $\begin{array}{l}\text { - Augmentation des charges fixes } \\
\text { et opérationnelles provoquée par } \\
\text { l'intensification de l'usage } \\
\text { de pratiques alternatives. } \\
\text { - Augmentation du temps de travail. } \\
\text { - Pénalités financières sanctionnant } \\
\text { le non-respect de la réglementation } \\
\text { environnementale. }\end{array}$ & $\begin{array}{l}\text { - Changement du contexte institutionnel (révision du } \\
\text { rôle des acteurs institutionnels, nouvelles politiques } \\
\text { agricoles, remise en cause à terme des aides publiques } \\
\text { accordées dans le cadre des MAET). } \\
\text { - Différenciation de la réglementation au sein de l'UE. } \\
\text { - Dépendance vis-à-vis des firmes de l'agrofourniture. } \\
\text { - Volatilité des prix des produits agricoles et } \\
\text { modification des marchés. }\end{array}$ \\
\hline
\end{tabular}

La plupart des risques et incertitudes trouvent leur origine davantage dans l'adoption d'un certain nombre de pratiques alternatives préconisées par la coopérative pour réduire l'utilisation des produits phytosanitaires que dans la contractualisation proprement dite, qui n'exige pour sa part que l'atteinte des objectifs IFT cible. Les pratiques préconisées comprennent la rotation des cultures, le zéro-labour, l'utilisation de la herse étrille et du binage, le faux semis et l'application raisonnée en fonction des modèles de prévision agropédo-climatiques. Si la plupart de ces nouvelles techniques culturales ont fait l'objet d'expérimentations et sont bien maîtrisées par certains groupes d'agriculteurs, elles restent bien souvent innovantes dans le contexte local. Leurs effets sur des systèmes de grandes cultures en plaine et en coteaux, zones agricoles jusque-là travaillées de manière conventionnelle, sont peu connus. Les craintes sont non seulement soulevées par rapport à une méconnaissance des techniques et nouveaux produits phytosanitaires, mais aussi par rapport à des effets visibles (envahissement des parcelles par des mauvaises herbes, érosion des sols). En conséquence, la relation directe aux techniques est difficile à cerner et pose question aux acteurs comme l'attestent les réflexions et les questionnements des agriculteurs et des conseillers que nous avons rencontrés sur le terrain et dont nous reproduisons ci-dessous quelques extraits tirés de nos entretiens : 
"Les nouveaux produits sont beaucoup plus concentrés, soi-disant moins dangereux. Mais on n'en est pas si sûr.»

(Agriculteur enquêté)

"C'est un peu l'inconnu. À un moment donné, il y aura sans doute plus de travail à cause des mauvaises herbes. »

(Agriculteur enquêté)

«Il y a un gros travail d'animation, de sensibilisation, surtout des anciennes générations, sur l'observation, la nuisibilité des adventices et sur le travail du sol. »

(Technicien de la coopérative enquêté)

\section{Traduction des risques et incertitudes en termes de coûts et bénéfices}

Avant de nous intéresser aux modes de coordination, qui sous-tendent le processus informationnel intervenant dans l'adoption des MAET, il nous paraît nécessaire de discuter de la traduction des risques et incertitudes identifiés en termes de coûts et de bénéfices. Bien souvent dans la littérature, les risques et les incertitudes sont appréhendés comme des contraintes ou encore comme un coût global à l'adoption. Ce point de vue doit être nuancé. En effet, lorsqu'ils relèvent du court terme, les risques et incertitudes peuvent se traduire de manière générale par un coût privé qui pèse dans la décision de contractualisation des agriculteurs. Cependant, sur le long terme, certains événements considérés comme risqués ou incertains peuvent engendrer un changement structurel positif au niveau du système de production ou encore produire des externalités positives. En conséquence, ces événements peuvent jouer un rôle central dans les processus d'apprentissage collectif et être à l'origine de gains cognitifs (Callon et al., 2001). Ainsi, dans le cas examiné ici, les situations de risque et l'incertitude peuvent être propices au développement de nouvelles techniques de production plus efficaces. Prenons l'exemple de l'augmentation des charges opérationnelles (recours à de nouveaux produits, pratiques plus exigeantes en temps, etc.) et fixes (investissement dans un pulvérisateur de précision, une herse étrille...) liée à l'adoption et à l'intensification des pratiques préconisées par la coopérative. La maîtrise d'une nouvelle technique (réglage de la herse étrille, choix de la rotation...) représente un coût privé sur le court terme. Toutefois, sur le long terme, l'emploi de ces techniques peut générer des bénéfices privés pour l'agriculteur: lissage des pics de travail, préservation de la santé de l'agriculteur, recherche de nouvelles pratiques, etc. Les agriculteurs que nous avons rencontrés sont conscients de ces enjeux, comme l'indique la remarque suivante de l'un d'entre eux :

« Je suis déjà engagé dans des réductions pour des raisons économiques, mais aussi conscient de l'importance de réduire pour plus tard. Je suis conscient des dangers des produits phytosanitaires. Je saigne parfois avec certains produits. [...] De toutes façons, les réductions seront obligatoires dans quelques années alors c'est bien de se préparer, surtout pour le fils qui va s'installer. »

(Agriculteur enquêté)

Sur le long terme, il est également indispensable de tenir compte des bénéfices collectifs résultant non seulement de la réduction des phytosanitaires proprement dite (restauration de la qualité de l'eau, maintien de l'équilibre du sol et de la biodiversité) mais aussi de l'apprentissage collectif (production de connaissances et constitution de réseaux grâce aux formes collectives de conseil). Ces externalités positives, difficilement évaluables a priori, ont en effet une incidence majeure dans la balance coûts/bénéfices du projet MAET dans son ensemble. Lors de notre enquête, l'un des techniciens de la coopérative a clairement exprimé les termes de cet enjeu : 
«La finalité de la démarche reste l'amélioration de la qualité de l'eau. Pour le technicien, l'eau est un enjeu prioritaire aux enjeux alimentaires. [...] Mon objectif est d'amener les agriculteurs à travailler autrement. "

(Technicien de la coopérative enquêté)

Le tableau 2 présente une typologie qualitative des agriculteurs enquêtés en fonction de leur degré d'aversion et de leur appréciation des coûts et bénéfices privés et collectifs liés aux événements identifiés au préalable comme risqués et/ou incertains. Ce degré d'aversion a été apprécié qualitativement à partir d'indicateurs habituellement utilisés, à savoir des indicateurs reflétant la mise en œuvre de stratégies de gestion ex-ante et ex-post des risques (diversification des ateliers et cultures, souscription à une assurance, investissements réalisés). Un système de notation (allant de + à +++ ) a été construit selon l'importance des stratégies de gestion des risques développées sur l'exploitation. Cette évaluation de l'aversion aux risques a été menée indépendamment de l'appréciation de la perception des coûts et bénéfices de l'adoption. Cette dernière renvoie aux réponses données par les agriculteurs quant à leur propre évaluation des coûts et bénéfices des changements techniques et organisationnels induits par l'adhésion à la MAET-DCE (une note moyenne allant de - à +++ , respectivement pour les agriculteurs qui ne relèvent aucun coûts ou bénéfices et pour ceux qui rapportent les coûts ou bénéfices les plus élevés).

Tableau 2. Typologie des agriculteurs en fonction de leur degré d'aversion et de leur appréciation des coûts et bénéfices liés aux événements risqués et incertains

\begin{tabular}{|c|c|c|c|c|}
\hline & $\begin{array}{c}\text { Type } 1 \\
\text { « Conservateurs » } \\
\text { Sont confiants } \\
\text { de leur maîtrise } \\
\text { technique }\end{array}$ & $\begin{array}{c}\text { Type } 2 \\
\text { « Prudents » } \\
\text { Se replient sur } \\
\text { des solutions } \\
\text { connues }\end{array}$ & $\begin{array}{c}\text { Type } 3 \\
\text { "Prudents » } \\
\text { Préfèrent } \\
\text { anticiper sur } \\
\text { l'avenir }\end{array}$ & $\begin{array}{c}\text { Type } 4 \\
\text { « Innovants » } \\
\text { Investissent et testent }\end{array}$ \\
\hline Nombre enquêtés & 2 & 3 & 3 & 9 \\
\hline $\begin{array}{l}\text { Caractéristiques } \\
\text { exploitation }\end{array}$ & $\begin{array}{l}\text { SAU variable } \\
\text { Proche de la } \\
\text { retraite } \\
\text { Un seul UTH } \\
\text { Convaincus par } \\
\text { leurs pratiques } \\
\text { " d'agriculture } \\
\text { raisonnée " }\end{array}$ & $\begin{array}{l}\text { SAU>100 ha } \\
45 \text { à } 50 \text { ans } \\
\text { Activités hors } \\
\text { exploitation } \\
\text { Contrainte } \\
\text { de temps forte }\end{array}$ & $\begin{array}{c}S A U<100 \text { ha } \\
45 \text { à } 50 \text { ans } \\
\text { Peu techniques } \\
\text { Un seul UTH } \\
\text { Anciens fidèles } \\
\text { à la coopérative }\end{array}$ & $\begin{array}{c}\text { SAU variable } \\
30 \text { à } 45 \text { ans } \\
\text { Activités diversifiées } \\
\text { (sur l'exploitation) } \\
\text { Plusieurs UTH } \\
\text { Expérience des } \\
\text { négociants } \\
\text { Expérience des CTE et } \\
\text { pratiques alternatives }\end{array}$ \\
\hline $\begin{array}{l}\text { Tendance à l'adoption } \\
\text { de nouvelles pratiques }\end{array}$ & non & oui/non & Oui & oui \\
\hline \multirow{2}{*}{$\begin{array}{c}\text { Degré d'aversion } \\
\text { Perception des coûts } \\
\text { privés }\end{array}$} & +++ & ++ & ++ & + \\
\hline & +++ & +++ & ++ & ++ \\
\hline \multirow{3}{*}{$\begin{array}{c}\text { Perception des coûts } \\
\text { collectifs } \\
\text { Perception } \\
\text { des bénéfices privés } \\
\text { Perception } \\
\text { des bénéfices collectifs }\end{array}$} & - & + & ++ & + \\
\hline & + & ++ & +++ & +++ \\
\hline & - & + & ++ & +++ \\
\hline
\end{tabular}


Cette typologie, même si elle repose pour l'instant sur un échantillon limité d'enquêtes, suggère que la différence observée entre les agriculteurs par rapport à l'adoption de nouvelles techniques serait moins liée à leur degré d'aversion qu'à leur perception des coûts et bénéfices de l'adoption. Un agriculteur présentant une forte aversion aux risques (type 1) sera moins prêt à adopter de nouvelles pratiques qu'un agriculteur ayant une faible aversion aux risques (type 4). Cependant, pour un même degré d'aversion aux risques, certains agriculteurs vont adopter la MAET car ils perçoivent davantage de bénéfices privés et collectifs que de coûts (type 3). Cette adoption est elle-même liée aux caractéristiques de l'exploitation et au parcours de l'agriculteur. Ce constat confirme d'ailleurs les résultats obtenus dans d'autres études qui ont évalué l'aversion aux risques (Tucker et Napier, 2001 ; Aubertot et al., 2007).

Nous notons par ailleurs que la majorité des agriculteurs sont davantage préoccupés par les coûts et bénéfices privés sur le court terme que par les bénéfices collectifs pouvant être dégagés sur le long terme. Lorsqu'ils mentionnent le long terme, ils prennent essentiellement en compte la nécessité d'anticiper les changements de la réglementation. Il existe, de ce fait, un décalage entre les objectifs à long terme de la MAET mis en avant par les techniciens de la coopérative et ceux reformulés par les agriculteurs contractants. Ce décalage traduit toute la difficulté liée à la mise en œuvre d'une mesure pour laquelle les coûts/bénéfices privés et collectifs ne pourront être connus que sur le long terme.

Il s'avère cependant que l'évolution des perceptions des agriculteurs vis-àvis des risques et des incertitudes permet de réduire la tension entre la recherche de la maximisation de l'utilité espérée et la promotion d'intérêts collectifs tels que l'amélioration de la qualité de la ressource en eau. En effet, comme nous avons pu l'observer dans nos entretiens, les agriculteurs sont d'autant plus enclins à intégrer des valeurs collectives dans leur choix d'action qu'ils ont le sentiment de disposer de solutions techniques pour surmonter les incertitudes générées par un changement de pratiques. Ainsi, lorsque c'est le cas, ces valeurs collectives deviennent des normes sociales intériorisées dont ils se servent pour porter, par exemple, un jugement sur les pratiques agricoles d'un voisin comme l'illustre l'énoncé suivant :

"J'ai un voisin qui a de la flotte, qui irrigue. Lui, il est au moins au 10 tour. Oui et puis, c'est pas de petits tours qu'il fait. Moi, je trouve que c'est gaspiller de l'eau.»

(Agriculteur enquêté)

Nous en venons à présent aux formes d'apprentissage et à leurs impacts sur la réduction des risques et des incertitudes.

\section{Les formes d'apprentissage et la réduction des risques et incertitudes}

Nous avons précisé précédemment (supra) que la principale originalité du dispositif MAET-DCE étudié repose sur l'offre par la coopérative d'un service de conseil facturé aux agriculteurs $25 € /$ ha. Pour les agriculteurs engagés dans la MAET-DCE, le coût de ce service de conseil est actuellement compensé par les aides qu'ils perçoivent de la part des pouvoirs publics. La coopérative envisage néanmoins de pérenniser ce dispositif de conseil payant au terme de la MAET. Elle compte pour cela convaincre les agriculteurs qu'un suivi régulier de leur exploitation permet, du fait de l'adoption des innovations techniques proposées, de réaliser d'importantes économies en intrants. C'est pourquoi le message véhiculé par le technicien spécialisé MAE de la coopérative est avant tout technique et 
concerne le travail du sol, les pratiques agronomiques de rotation, le raisonnement des traitements en fonction des prévisions, etc. La coopérative a d'ailleurs rendu le conseil obligatoire pour tout contractant car elle considère que «la réduction des interventions phytosanitaires demande une technicité supérieure aux pratiques habituelles si l'objectif est de maintenir rendement et qualité » (Projet MAET-DCE, document interne, 17 février 2010).

En définitive, l'introduction d'un service de conseil payant contribue à modifier progressivement les relations entre les agriculteurs et la coopérative et à renforcer les attentes et les engagements réciproques entre les deux types d'acteurs. Ainsi, dans le cadre actuel de la MAET, la coopérative recourt à une gamme étendue de formes organisationnelles de conseil: visites individuelles classiques, mais également actions d'animation collective (visites de champs d'essai, démonstrations de matériels), prestations de conseil à distance avec la mise en place récente d'un système informatisé d'alerte météorologique, etc.

Le tableau 3, recense en colonne les différentes formes d'information et d'apprentissage auxquelles ont recours les agriculteurs de notre échantillon : conseils émanant de la coopérative, mais provenant également d'autres acteurs ou d'autres sources (voisins, Chambre d'agriculture, réseau technique, revus techniques). La dernière ligne du tableau indique le degré d'importance (noté de +++ à -) que ces agriculteurs attribuent à chacune des formes d'information et d'apprentissage recensées. Cette importance a été appréciée en faisant la moyenne des notes attribuées par les agriculteurs interrogés à ce sujet, sur la base d'une échelle de type Likert avec 5 modalités.

Le tableau 3 permet de mettre en évidence que les agriculteurs accordent une importance similaire au conseil fourni par la coopérative et aux échanges informels entre agriculteurs. Il s'agit de deux formes de «learning by others » dont l'efficacité a été démontrée par de nombreuses études (Hiebert, 1974 ; Feather et Amacher, 1994 ; Learn Group, 2000). La confiance vis-à-vis de l'information provenant de la coopérative s'explique principalement par le capital relationnel dont elle bénéficie de la part des agriculteurs. Ce capital a été élaboré au cours du temps : pilotage de démarches de qualité et de CTE collectifs. Kephaliacos et al. (2006) ont d'ailleurs montré que l'existence d'actions collectives antérieures, en contribuant à la construction d'un capital «confiance et connaissance », permet de réduire significativement les coûts de transaction et de favoriser l'engagement dans des dispositifs contractuels qui peuvent paraitre a priori contraignants pour l'agriculteur. D'autres transactions développées par la coopérative avec les organismes publiques (DDEA, DDASS) ont aussi permis de renforcer ce capital relationnel. En se posant comme interlocuteur privilégié et reconnu des agriculteurs, la coopérative a, en effet, réussi à légitimer le projet MAET et les messages techniques associés.

Nous assimilons cette relation entre la coopérative et les contractants à une transaction de marchandage de type «win-win». Entre ces deux catégories d'acteurs, s'opère un transfert de droits de propriété qui conduit à la constitution et à l'usage d'un certain nombre de biens club : connaissances techniques, valorisation collective de la qualité (produits et environnementale) et autres externalités de réseau. Ce transfert participe à réduire non seulement les risques classiquement perçus par les agriculteurs (en particulier les risques de production relatifs à la mauvaise maîtrise de nouvelles techniques), mais également les incertitudes liées aux changements attendus de politiques agricoles et non pris en compte par les termes actuels du contrat : 
RECHERCHES

Geneviève NGUYEN, Jean-Pierre DEL CORSO, Charilaos KEPHALIACOS, Henri TAVERNIER

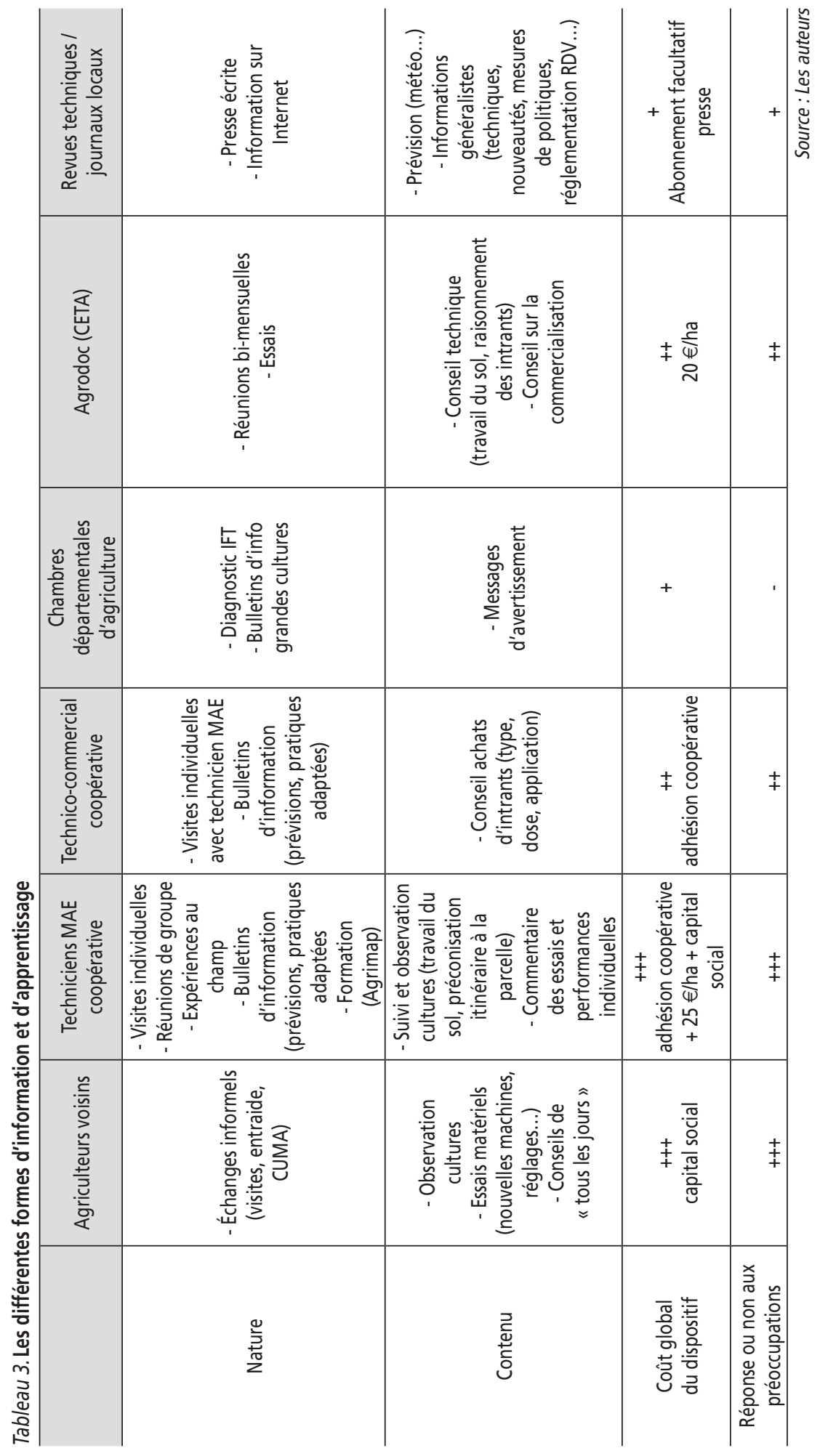


«Un bio était venu montrer son matériel. C'était très bien, même si on n'avait pas eu le temps d'aborder tous les points négatifs et positifs. On met ça en place chez nous puis on se fera un avis. "

(Agriculteur enquêté)

«À chaque fois que la coopérative a poussé les agriculteurs dans des démarches, comme les CTE, le blé Galibier, cela a toujours été positif économiquement et techniquement. Elle nous permet d'avoir une longueur d'avance sur les autres agriculteurs par rapport à des réductions qui vont devenir obligatoires. »

(Agriculteur enquêté)

La relation d'apprentissage construite au sein de groupes informels d'agriculteurs peut également être caractérisée de transaction de marchandage. Cependant, si cette transaction contribue à la production d'externalités de réseau, elle ne permet pas nécessairement la production de biens environnementaux locaux. En effet, à la différence du conseil technique fourni par la coopérative, dont l'objectif est d'amener les agriculteurs à adopter globalement des pratiques alternatives avec des objectifs de résultat déterminés, l'information échangée de manière informelle entre agriculteurs concerne surtout des points techniques très précis : observation du résultat d'un faux-semis sur l'envahissement des parcelles par des mauvaises herbes, test de réglage d'un matériel, etc. Ces échanges ont pour but principal de réduire les coûts de production tout en maintenant le niveau de rendement et en contrôlant les risques et les incertitudes de nature agronomique. Ils ne visent pas directement l'amélioration de l'état des ressources environnementales et n'agissent donc pas sur les incertitudes de nature économique de la même manière que le conseil technique de la coopérative.

$$
* *
$$

En définitive, au-delà de sa complexité, le dispositif multi-partenarial mis en place se révèle être d'une grande cohérence par rapport à l'objectif fixé de réduction des produits phytosanitaires. Certes, la coopérative vend des intrants agricoles, mais son initiative de porter un projet en faveur de l'environnement est légitimée par ses actions antérieures (CTE collectifs) et en cours (filière biologique) et par le capital social qu'elle a su construire et accumuler. En se positionnant comme intermédiaire entre, d'une part, les organismes publics et les firmes d'agrofourniture, et d'autre part, les agriculteurs, elle permet d'évacuer un certain nombre d'incertitudes de nature économique et institutionnelle, et de recadrer le projet MAET-DCE autour d'un dispositif d'apprentissage collectif accompagné par un discours avant tout technique qui rassure les agriculteurs.

L'action de la coopérative est notamment au centre de la création conjointe de plusieurs catégories de biens via plusieurs types de coordinations entre les acteurs concernés, individuels, collectifs ou d'organismes privés ou publics. Ce type de production jointe va au-delà des phénomènes déjà identifiés dans la littérature économique à propos de la multifonctionnalité de l'agriculture et la gestion des ressources naturelles en général. Ils concernent plus particulièrement les processus d'apprentissage et la création multi-partenariale de connaissances nécessaires au changement technique mais aussi institutionnel qui est proposé (par exemple, les démarches volontaires) ou imposé (par exemple, la conditionnalité des aides de la PAC) dans une optique de développement durable.

Du point de vue économique et à partir du cas étudié, nous avons ainsi proposé une nouvelle interprétation des phénomènes pouvant avoir lieu lors de la mise en œuvre 
des politiques environnementales visant le changement des pratiques de production et touchant de fait un ensemble de partenaires comme ceux intervenant dans une filière de production agroalimentaire. Il reste bien sûr la question de la généralisation de ces résultats dans la mesure où ils retracent une situation de terrain relativement unique en France. Il est peut-être intéressant de noter à ce propos qu'un élément constitutif de l'expérience étudiée est son inscription dans une histoire locale (Kephaliacos et Robin, 2004 ; Kephaliacos et al., 2006). De ce point de vue, le cas étudié présente une valeur en soi dans la mesure où il peut permettre de dresser une liste d'ingrédients nécessaires pour que « ça fonctionne » ou que «ça puisse commencer à fonctionner ». Parmi ces ingrédients, la confiance entre acteurs et le «pouvoir d'actualisation des croyances » (Bromley, 2008) détenu par un acteur (ici la coopérative, du fait de la légitimité dont elle dispose auprès des agriculteurs) semblent jouer un rôle essentiel.

Sur la base de ces considérations, il est ainsi possible de proposer une nouvelle conception de l'activité de conseil dans laquelle nous pouvons distinguer quatre facettes originales. Premièrement, nos résultats mettent en avant l'évolution d'une logique de prescription à une logique d'accompagnement, où le conseil est adapté à chaque situation et basé sur un co-diagnostic des problèmes et une coconstruction des solutions. Cet aspect entre en résonance avec des travaux récents réalisés sur le conseil en agriculture (Albaladéjo et al., 2010 ; Rémy et al., 2006). Deuxièmement, ce conseil combine à la fois une dimension agronomique forte à une dimension environnementale et économique. Il contribue ainsi à accroître l'efficacité de politiques publiques visant une meilleure prise en compte des liens entre agricultures et environnement. Troisièmement, en se recentrant sur les aspects techniques et organisationnels, ce conseil facilite la transformation d'incertitudes perçues en risques perçus. En cela, il favorise une plus grande acceptation, par les agriculteurs, du changement en cours de la régulation publique dans le secteur agricole. Enfin, cette nouvelle conception du conseil est de nature à renforcer la légitimité de certains interlocuteurs professionnels des agriculteurs, parmi lesquels les coopératives. En effet, comme nous l'avons vu dans cet article, ces dernières peuvent s'appuyer sur leur crédibilité acquise dans le domaine technique pour convaincre les agriculteurs de la pertinence de certaines innovations agronomiques. C'est à ce titre qu'elles peuvent être appelées à jouer un rôle déterminant dans le processus de changement institutionnel et technique conduisant à la promotion du développement durable dans le secteur agricole.

\section{RÉFÉRENCES BIBLIOGRAPHIQUES}

Albaladejo C., Auricoste C., Barthe L., Couix N., Duvernoy I., Girard N., Gross H., Labatut J., Lenormand P. (2010). Le conseil agricole au carrefour du développement sectoriel et du développement territorial : accompagner l'activité agricole en situation. Communication au séminaire Innovation and Sustainable Development in Agriculture and Food (ISDA), 28 juin au $1^{\mathrm{er}}$ juillet, Montpellier, $12 \mathrm{p}$.
Aubertot J.-N., Barbier J.-M., Carpentier A., Gril J.-N., Guichard L., Lucas P., Savary S., Voltz M. (eds.) (2007). «Pesticides, agriculture et environnement: réduire l'utilisation des pesticides et en limiter les impacts environnementaux. Expertise scientifique collective Inra-Cemagref », Paris, Quae.

Binswanger H. P. (1980). Attitudes toward risk: Experimental measurement in rural India. 


\section{Pratiques agricoles pour la réduction des produits phytosanitaires}

American Journal of Agricultural Economics, $\mathrm{n}^{\circ} 62$, p. 395-407.

Brives H. (2006). Les conseillers agricoles et l'environnement: quelles compétences? In Rémy J., Brives H., Lémery B. (eds.), «Conseiller en agriculture », Paris, Éditions Inra, coll. « Sciences en partage ».

Bromley D. W. (2006). Sufficient Reason. Volitional Pragmatism and the Meaning of Economic Institutions. Princeton (USA), Princeton University Press.

Bromley D. W. (2008). Volitional pragmatism. Ecological Economics, n ${ }^{\circ} 68$ (1-2), p. 1-13.

Callon M. (1999). La sociologie peut-elle enrichir l'analyse économique des externalités ? Essai sur la notion de cadrage-débordement. In Mairesse D. (ed.), «Innovations et performances. Approches interdisciplinaires », Paris, Éditions de l'École des Hautes Études en Sciences Sociales, p. 399-431.

Callon M., Lascoumes P., Barthe Y. (2001). Agir dans un monde incertain. Essai sur la démocratie technique. Paris, Seuil.

Commons J. R. (1931). Institutional Economics. American Economic Review, $\mathrm{n}^{\circ} 21$, p. 57-648.

Feather P. M., Amacher G. S. (1994). Role on information in the adoption of best management practices for water quality improvement. Agricultural Economics, $\mathrm{n}^{\circ} 11$, p. 70-159.

Ghali B., Ridier A., Kephaliacos Ch., Nguyen G. (2009). Impact of voluntary measures on farm's income and labour management: The case study of a « Test-Action » in a river basin of South Western France. Communication à la «Troisième Journée de Recherches en Sciences Sociales » Montpellier, SFER, 9-11 décembre, $20 \mathrm{p}$.

Godard O. (1994). Le développement durable, paysage intellectuel. Nature, Sciences, Sociétés, vol. $2, \mathrm{n}^{\circ} 4$, p. 309-322.

Godard O. (2003). Le principe de précaution comme norme de l'action publique, ou la proportionalité en question. Revue économique, vol. 54, p. 1245-1276.

Hardaker J. B. (2000). Some issues in dealing with risks in agriculture. WP No.2000-3, Working Paper series in Agricultural and Resource Economics, Graduate School of
Agricultural and Resource Economics, University of New England, 18 p.

Hardaker J. B., Huirne R. B. M., Anderson J. R. (2004). Coping with Risk in Agriculture, CAB International, Wallingford (UK).

Hiebert L. D. (1974). Risk, learning, and the adoption of fertilizer responsive seed varieties. American Journal of Agricultural Economics, vol. $56, \mathrm{n}^{\circ} 4$, p. 764-68.

Just R. E., Pope R. D. (1979). On the relationship of input decisions and risk. In Roumasset J. A., Boussard J. M, Singh I. (eds.), «Risk, uncertainty and Agricultural Development », New York, Agricultural Development Council.

Kephaliacos Ch., Robin P. (2004). Implementing environmental quality standards via collective projects in the French CTE procedure. International Journal of Agricultural Resources, Governance and Ecology, n³, p. 1-10.

Kephaliacos Ch., Nguyen G., Robin P. (2006). Formes de coordination et politiques publiques: Quelles articulations dans une perspective d'ancrage territorial de l'agriculture en France. Revue canadienne des sciences régionales, vol. 29, numéro spécial « Politique publique et espace rural», p. 119-140.

LEARN Group (2000). Cow Up A Tree. Knowing and Learning for Change in Agriculture. Case Studies from Industrialized Countries. Paris, INRA.

Ministère de l'Ecologie et du Développement Durable (MEDD) (2005) Agriculture et environnement. Rapport à la Commission des comptes et de l'économie de l'environnement. Paris, La Documentation Française.

Pindyck R. S. (2007). Uncertainty and Environmental Economics. Review of Environmental Economics and Policy, vol. 1, $\mathrm{n}^{\circ} 1$, p. 45-56.

Rémy J., Brives H., Lémery B. (eds.) (2006). Conseiller en agriculture. Paris, INRA, coll. « Sciences en partage».

Tucker M., Napier T. L. (2001). Determinants of perceived agricultural chemical risk in three watersheds in the Midwester United States. Journal of Rural Studies, $\mathrm{n}^{\circ} 17$, p. 219-233. 
RECHERCHES

Geneviève NGUYEN, Jean-Pierre DEL CORSO, Charilaos KEPHALIACOS, Henri TAVERNIER

Vatn A. (2005). Institutions and the Environment. Cheltenham, Edward Elgar.

Vila C. (2009). La mise en ouvre négociée d'une mesure agri-environnementale portée par une coopérative agricole: un cas d'étude sur le bassin versant de la Gimone. Mémoire de Master 2, Institut Professionnel Universitaire de Sociologie Appliquée, Université Toulouse le Mirail, 58 p. 